\title{
Large $\operatorname{BR}(h \rightarrow \tau \mu)$ in the MSSM?
}

\section{Daniel Aloni, Yosef Nir and Emmanuel Stamou}

Department of Particle Physics and Astrophysics, Weizmann Institute of Science, Rehovot 7610001, Israel

E-mail: daniel.aloni@weizmann.ac.il, yosef.nir@weizmann.ac.il, emmanuel.stamou@weizmann.ac.il

Abstract: We study how large the rate of the lepton-flavor violating Higgs decay $h \rightarrow \tau \mu$ can be in the (R-parity conserving) MSSM. We make no assumptions, such as universality or alignment, about the flavor structure of the MSSM. We only assume that all couplings and, in particular, the trilinear scalar ones, are perturbative. We take into account lower bounds on the bino and slepton masses from $\tau \rightarrow \mu \gamma$ and $h \rightarrow \gamma \gamma$ as well as upper bounds on the trilinear scalar couplings from the requirement that the global minimum is not charge breaking. We find that in highly fine-tuned regions of parameter space, the ratio $\mathrm{BR}(h \rightarrow \tau \mu) / \mathrm{BR}(h \rightarrow \tau \tau)$ can be enhanced by about three orders of magnitude above the estimate from naive dimensional analysis, but still about two orders of magnitude below the present bound. Thus, if $h \rightarrow \tau \mu$ is experimentally established to be close to present bounds, the MSSM will be excluded.

KEYWORDS: Supersymmetry Phenomenology

ArXiv EPrint: 1511.00979 


\section{Contents}

1 Introduction 1

2 The theoretical framework 2

3 LFV from the $A^{E}$ terms $\quad 5$

$\begin{array}{lll}4 & \text { LFV from the } \tilde{m}_{L}^{2} \text { terms } & 7\end{array}$

5 Charge breaking minima $\quad 10$

6 Conclusions 12

$\begin{array}{ll}\text { A Field renormalization } & 14\end{array}$

$\begin{array}{ll}\text { B Constraints in the } \cos (\beta-\alpha)-\tan \beta \text { plane } & 15\end{array}$

$\begin{array}{ll}\mathrm{C} \tau \rightarrow \mu \gamma & 16\end{array}$

$\begin{array}{ll}\mathrm{D} h \rightarrow \gamma \gamma & 17\end{array}$

\section{Introduction}

The first direct searches for the lepton-flavor violating (LFV) Higgs decay $h \rightarrow \tau \mu$ were carried out by the CMS and ATLAS collaborations [1,2] yielding the upper bounds:

$$
\mathrm{BR}(h \rightarrow \tau \mu)<\left\{\begin{array}{l}
1.51 \times 10^{-2} \quad \mathrm{CMS}, \\
1.85 \times 10^{-2} \text { ATLAS }
\end{array}\right.
$$

and the ranges:

$$
\mathrm{BR}(h \rightarrow \tau \mu)=\left\{\begin{array}{c}
\left(8.4_{-3.7}^{+3.9}\right) \times 10^{-3} \quad \text { CMS } \\
(7.7 \pm 6.2) \times 10^{-3} \text { ATLAS }
\end{array}\right.
$$

The $h \rightarrow \tau \mu$ decay has several aspects that are worth emphasizing:

- It violates the lepton-flavor symmetry $\mathrm{U}(1)_{\mu} \times \mathrm{U}(1)_{\tau}$, which is an accidental symmetry of the Standard Model (SM).

- It is a flavor changing neutral current (FCNC) process.

- It violates the prediction that the Yukawa matrix is proportional to the mass matrix, $Y^{E} \propto M_{E}$, which applies at the tree level to all models of Natural Flavor Conservation (NFC). 
Due to these three aspects, an observation of $h \rightarrow \tau \mu$ in present experiments will have far reaching implications.

In this work we ask whether an $h \rightarrow \tau \mu$ decay rate close to the near future sensitivity of the LHC experiments, $\mathrm{BR}(h \rightarrow \tau \mu)=\mathcal{O}(0.01)$, can be accounted for by the minimal supersymmetric standard model (MSSM). The branching ratio depends on the total width of the Higgs, which is experimentally unknown and which, within the MSSM, depends on the entire supersymmetric spectrum. To avoid the dependence on sectors unrelated to LFV and on experimentally yet-unconstrained observables, we consider the ratio of branching ratios,

$$
R_{\tau \mu / \tau \tau} \equiv \frac{\mathrm{BR}(h \rightarrow \tau \mu)}{\operatorname{BR}(h \rightarrow \tau \tau)},
$$

which is independent of the total width. In particular, $R_{\tau \mu / \tau \tau}$ is insensitive to the spectrum of the colored particles. By combining $h \rightarrow \tau \tau$ and $h \rightarrow \tau \mu$ data we obtain the experimentally allowed range for the ratio of branching ratios

$$
0.07(0.01) \lesssim R_{\tau \mu / \tau \tau} \lesssim 0.21(0.31) \quad \text { at } 68.3 \%(95 \%) \text { C.L. }
$$

For this bound we assumed a parabolic $\chi^{2}$, i.e. gaussian errors, and profiled over $\operatorname{BR}(h \rightarrow$ $\tau \tau)$ to obtain the C.L. interval on the ratio. We thus focus on whether the MSSM can account for $R_{\tau \mu / \tau \tau} \gtrsim 0.1$.

The LHC measurements of the $h \rightarrow \tau \mu$ decay rate and their implications for new physics have been discussed in the literature within various theoretical frameworks [3-24]. In particular, previous studies of $h \rightarrow \tau \mu$ within the supersymmetric framework have been carried out in refs. [25-28]. In these studies the emphasis was on identifying the range of $\mathrm{BR}(h \rightarrow \tau \mu)$ that corresponds to generic points in the parameter space. Indeed, we confirm that for generic supersymmetric parameters, $\operatorname{BR}(h \rightarrow \tau \mu)$ is several orders of magnitude below the present experimental sensitivity, as found in these previous works. We, however, are interested to learn whether, if $\operatorname{BR}(h \rightarrow \tau \mu)=\mathcal{O}(0.01)$ is established at the LHC, the MSSM will be not just disfavored but actually excluded. To answer this question, we allow the parameters to be highly fine-tuned and far from generic.

The structure of this paper is as follows. In section 2 we present our theoretical framework. In sections 3 and 4 we obtain the largest possible $R_{\tau \mu / \tau \tau}$ that can arise from LFV from the $A$-terms and the slepton masses-squared, respectively, taking into account bounds from $\tau \rightarrow \mu \gamma$ and $h \rightarrow \gamma \gamma$ and from perturbativity. In section 5 we require, in addition, that the electroweak symmetry breaking minimum is the global one and, in particular, that there is no deeper minimum that is charge breaking. We conclude in section 6. Supplementary material is delegated to the appendices.

\section{The theoretical framework}

We consider the minimal supersymmetric SM. We assume R-parity conservation (RPC), but make no assumptions, such as universality or alignment, about the supersymmetric mass spectrum and mixing pattern. Examining the MSSM in view of the three points emphasized above, we make the following observations: 
- Lepton flavor is not an accidental symmetry of the MSSM.

- Within the MSSM, FCNCs are always loop mediated.

- The R-parity even scalar sector of the MSSM is a two Higgs doublet model (2HDM) with NFC type-II.

We now elaborate on each of these three features of the MSSM.

The supersymmetric part of the MSSM Lagrangian is minimally flavor violating: the only supersymmetric sources of flavor violation are the Yukawa matrices of the SM. Therefore, this part of the Lagrangian has the same accidental $\mathrm{U}(1)_{e} \times \mathrm{U}(1)_{\mu} \times \mathrm{U}(1)_{\tau}$ symmetry as the SM. However, this is in general not the case for the soft supersymmetry breaking terms. They have three sources of LFV:

$$
\mathcal{L}_{\mathrm{MSSM}}^{\mathrm{LFV}}=-\tilde{m}_{L_{i j}}^{2} \tilde{L}_{i}^{\dagger} \tilde{L}_{j}-\tilde{m}_{R_{i j}}^{2} \tilde{\bar{E}}_{i}^{\dagger} \tilde{\bar{E}}_{j}-\left(A_{i j}^{E} h_{d} \tilde{L}_{i} \tilde{\bar{E}}_{j}+\text { h.c. }\right) .
$$

Here $\tilde{L}_{i}$ are the $\mathrm{SU}(2)$-doublet sleptons, $\tilde{\bar{E}}_{i}$ are the $\mathrm{SU}(2)$-singlet charged sleptons, and $h_{d}$ is the $Y=-1 / 2$ Higgs doublet; $\tilde{m}_{L}^{2}$ is the $3 \times 3$ mass-squared matrix for the doublet sleptons, $\tilde{m}_{R}^{2}$ is the $3 \times 3$ mass-squared matrix for the singlet sleptons, and $A^{E}$ is the $3 \times 3$ matrix of trilinear scalar couplings. Throughout this work we follow the conventions of ref. [29].

Since either $h \rightarrow \tau \mu$ or $h \rightarrow \tau e$, but not both, can be large [3], we decouple in what follows the selectron, and consider only the $2 \times 2 \mu-\tau$ block of each of the three matrices.

In addition to $\mathcal{L}_{\mathrm{MSSM}}^{\mathrm{LFV}}$, the following superpotential terms, involving the Higgs $\left(H_{u}, H_{d}\right)$ and lepton $(L, \bar{E})$ superfields, are relevant to our study:

$$
W_{H, L, E}=\mu H_{u} H_{d}+Y^{E} H_{d} L \bar{E} .
$$

In the charged lepton mass basis, $Y^{E}=\operatorname{diag}\left(y_{e}, y_{\mu}, y_{\tau}\right)$.

Two additional parameters that affect our results are the angles $\beta$ and $\alpha$. At the tree level they are traditionally defined as $\tan \beta=v_{u} / v_{d}$ (where $v_{u, d}=\left\langle H_{u, d}\right\rangle$ ), and $\alpha$ the rotation angle from the $\left(h_{d}, h_{u}\right)$ basis to the mass basis of the neutral CP-even Higgs mass eigenstates $(h, H)$. However, we consider loop corrections, where subtleties arise in the definitions of these parameters. The definition that we use (and is particularly convenient for our purposes) is given in eq. (B.1). In what follows, we use the notations $t_{\phi} \equiv \tan \phi$, $c_{\phi} \equiv \cos \phi$, and $s_{\phi} \equiv \sin \phi$ for the various angles that are relevant to our analysis.

Within the RPC MSSM, FCNC processes in general, and the $h \rightarrow \tau \mu$ decay in particular, get no tree-level contributions. The leading one-loop diagrams that contribute to this process are presented in figure 1 . In these diagrams, $\tilde{\ell}$ stands for the charged sleptons, $\tilde{\nu}$ for the sneutrinos, $\tilde{B}$ for the bino and $\tilde{W}$ for the wino.

The diagrams of figure 1 a are proportional to $y_{\tau} \times \sin 2 \theta \times \frac{\alpha}{4 \pi}$, where $\theta$ is the smuonstau mixing angle. In addition, this contribution is proportional to a loop function that depends on ratios of sparticle mass parameters and is, at most, of $\mathcal{O}(1)$. The electroweak loop factor of $\frac{\alpha}{4 \pi}$ suppresses the amplitude by three orders of magnitude with respect to the tree level $h \rightarrow \tau \tau$ decay. Thus, these diagrams cannot generate $R_{\tau \mu / \tau \tau} \gtrsim 0.1$, and we do not consider them any further. 


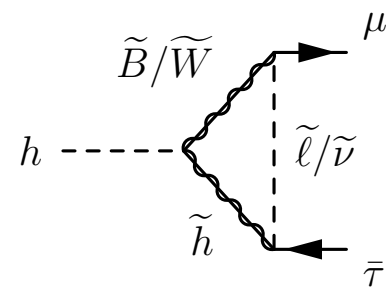

(a)

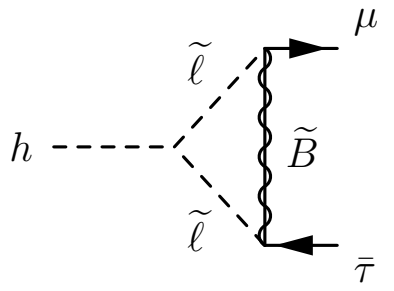

(b)

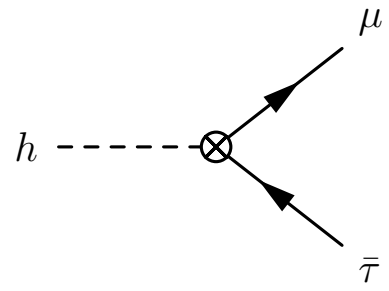

(c)

Figure 1. Diagrams contributing to the one-loop amplitude for $h \rightarrow \tau \mu$. $\otimes$ depicts the flavor off-diagonal counterterm from the field renormalization $\delta Z_{\tau \mu}$.

The diagrams of figure $1 \mathrm{~b}$ involve a trilinear scalar coupling. We distinguish two cases:

1. The trilinear scalar coupling arises from the supersymmetric terms $\mu Y^{E}$. This case has two important features. First, the source of LFV has to be either $\left(\tilde{m}_{L}^{2}\right)_{\mu \tau}$ or $\left(\tilde{m}_{R}^{2}\right)_{\mu \tau}$. Second, the relevant Higgs field is $h_{u}$, while the tree level tau Yukawa coupling involves $h_{d}$. In the limit of light 2HDM and heavy supersymmetry, the leading effect to $h \rightarrow \tau \mu$ arises from the misalignment between the vacuum expectation value and the light mass eigenstate and is therefore proportional to $c_{\beta-\alpha}$. Similarly to the diagrams of figure 1a, this contribution to $R_{\tau \mu / \tau \tau}$ is proportional to $\left[\sin 2 \theta \frac{\alpha}{4 \pi}\right]^{2}$. In this case, however, the contribution is proportional to the ratio of the dimensionful parameter $\mu$ and the bino or slepton mass. This factor can provide some enhancement.

2. The trilinear scalar coupling comes from the $A^{E}$ matrix. Now, the source of LFV can be the trilinear coupling itself, namely $A_{\mu \tau}^{E}$ or $A_{\tau \mu}^{E}$. Different from the previous case, the relevant Higgs field is $h_{d}$, the same as the one that has the diagonal tree-level coupling $y_{\tau}$. This contribution is, in general, not proportional to $y_{\tau}$. Nevertheless, if the mass scale of the sleptons and/or the bino is somewhat heavier than the electroweak scale, $m_{\mathrm{SUSY}}>v$, this contribution is suppressed by $v^{2} / m_{\text {SUSY }}^{2}$. This decoupling behavior is clear because in this case in the limit of heavy SUSY there is a single Higgs doublet so $h \rightarrow \tau \mu$ is mediated by the dimension-six operator $\frac{\lambda_{i j}}{m_{\text {SUSY }}^{2}} H^{3} \bar{L}_{i} E_{j}$.

In the next two sections we present how to maximize $h \rightarrow \tau \mu$ in each of those cases, taking into account relevant experimental bounds and perturbativity. The $A$-term case is analyzed in section 3 and the slepton mass-squared case in section 4 . The consequences of requiring that the global minimum is not charge breaking are analyzed, for both cases, in section 5 .

The diagram of figure $1 \mathrm{c}$ corresponds to the finite flavor off-diagonal counterterm related to the field renormalization $\delta Z_{\tau \mu}$. It ensures that lepton fields are canonically normalized and it is essential to include it in order to have the correct decoupling behavior. Its computation is outlined in appendix A.

Finally, for the calculation of the ratio $R_{\tau \mu / \tau \tau}$, we also need the tree-level contribution

$$
|\mathcal{M}(h \rightarrow \tau \tau)|^{2}=2 m_{h}^{2}\left(\frac{m_{\tau}}{v} \frac{s_{\alpha}}{c_{\beta}}\right)^{2} .
$$



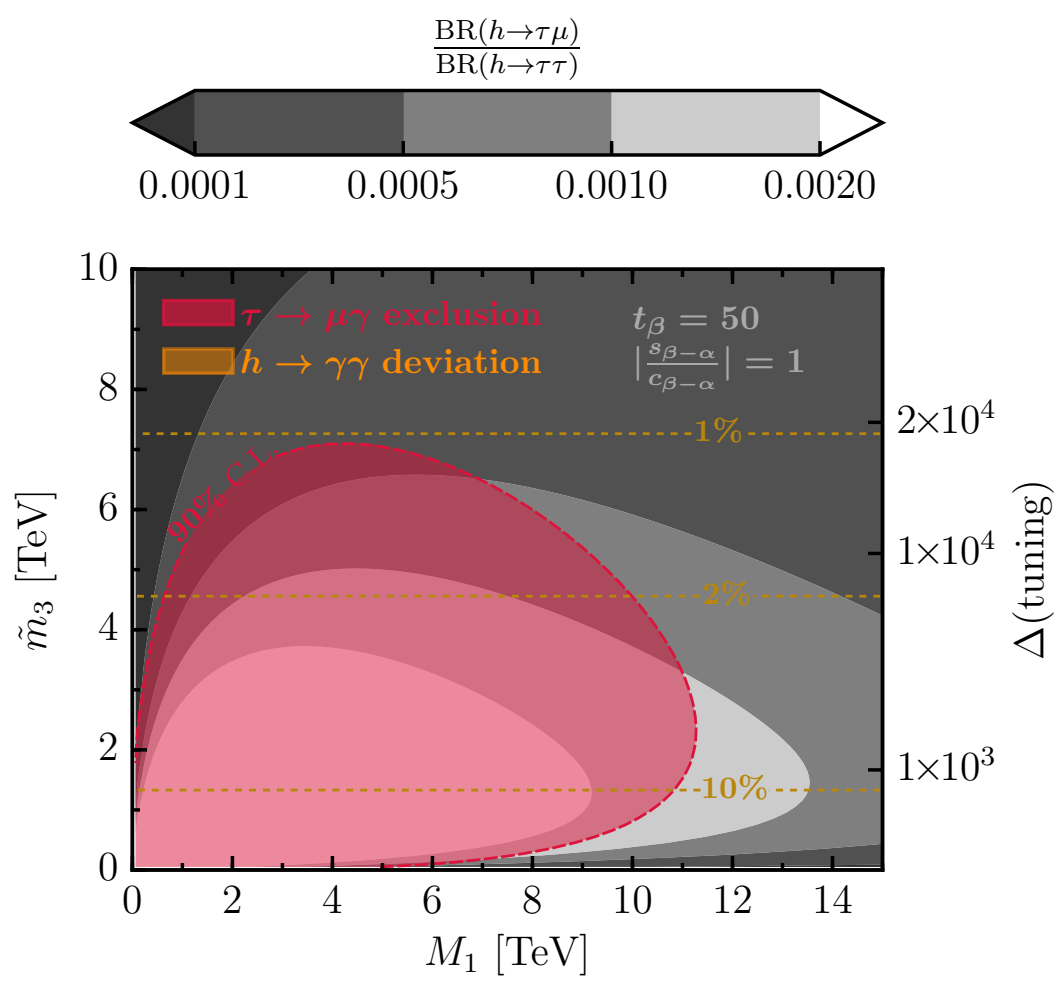

Figure 2. Contours of $R_{\tau \mu / \tau \tau}$ in the $M_{1}-\tilde{m}_{3}$ for the case of LFV from the A-term $A_{\mu \tau}$. The value of the A-term is the maximal allowed by perturbativity and satisfies vacuum stability constraints; the associated tuning is indicated on the right $y$-axis. The red region in dashed lines is excluded by the bound on $\tau \rightarrow \mu \gamma$. Orange horizontal lines indicate the deviation of the partial $h \rightarrow \gamma \gamma$ width with respect to the SM value.

Above we neglected terms that are suppressed by additional powers of external fermion masses. Our choice for defining $\alpha$ and $\beta$ at higher orders in perturbation theory, given in appendix B, ensures that eq. (2.3) remains valid also at the loop level.

\section{$3 \quad$ LFV from the $A^{E}$ terms}

Consider the case that the $\tilde{m}_{L}^{2}$ and $\tilde{m}_{R}^{2}$ matrices are diagonal, and the only source of LFV is the $A^{E}$ matrix. Both $A_{\mu \tau}^{E}$ and $A_{\mu \tau}^{E}$ contribute to the $h \rightarrow \tau \mu$ decay. The analysis is simplified if the $\tilde{\tau}_{L}^{\dagger} \tilde{\tau}_{R}$ entry in the mass-squared matrix can be neglected. Then, the $4 \times 4$ mass-squared matrix decomposes into two $2 \times 2$ blocks. For concreteness, we analyze the $\tilde{\mu}_{L}-\tilde{\tau}_{R}$ block. The analysis of the $\tilde{\tau}_{L}-\tilde{\mu}_{R}$ block is similar. The relevant part of the slepton mass-squared matrix has the following form:

$$
\tilde{\mathcal{M}}^{2}=\left(\begin{array}{cc}
\tilde{m}_{\mu_{L}}^{2} & \frac{v_{d} A_{\mu \tau}}{\sqrt{2}} \\
\frac{v_{d} A_{\mu \tau}}{\sqrt{2}} & \tilde{m}_{\tau_{R}}^{2}
\end{array}\right)
$$


The mixing angle $\theta$, which rotates from the interaction basis $\left(\tilde{\mu}_{L}, \tilde{\tau}_{R}\right)$ to the mass basis $\left(\tilde{\ell}_{3}, \tilde{\ell}_{2}\right)$, is given by

$$
\tan 2 \theta=\frac{\sqrt{2} v_{d} A_{\mu \tau}}{\tilde{m}_{\tau_{R}}^{2}-\tilde{m}_{\mu_{L}}^{2}}
$$

In the mass basis, the trilinear scalar couplings are given by

$$
\mathcal{L}_{h \tilde{\ell} \tilde{\ell}}=\frac{A_{\mu \tau} s_{\alpha}}{\sqrt{2}} h\left[s_{2 \theta}\left(\tilde{\ell}_{2}^{*} \tilde{\ell}_{2}-\tilde{\ell}_{3}^{*} \tilde{\ell}_{3}\right)+c_{2 \theta}\left(\tilde{\ell}_{2}^{*} \tilde{\ell}_{3}+\tilde{\ell}_{3}^{*} \tilde{\ell}_{2}\right)\right]
$$

and the bino-lepton-slepton couplings by

$$
\mathcal{L}_{\tilde{B} \tilde{\ell} \ell}=\frac{g^{\prime}}{\sqrt{2}} \tilde{B}\left(s_{\theta} \tilde{\ell}_{2}^{*}+c_{\theta} \tilde{\ell}_{3}^{*}\right) \mu_{L}-g^{\prime} \sqrt{2} \tilde{B}\left(c_{\theta} \tilde{\ell}_{2}-s_{\theta} \tilde{\ell}_{3}\right) \bar{\tau}_{R}+\text { h.c. }
$$

To zeroth order in the expansion of external momenta over SUSY masses, we obtain the following contributions to the decay amplitude of $h \rightarrow \tau^{+} \mu^{-}$:

$$
\begin{aligned}
& \text { figure 1c }=-i \frac{\alpha M_{1} s_{2 \theta} s_{\alpha}}{8 \pi c_{W}^{2} v c_{\beta}}\left(x_{2}-x_{3}\right) I_{3}\left(1, x_{2}, x_{3}\right) P_{R}, \\
& \text { figure } 1 \mathrm{~b}=i \frac{\alpha A_{\mu \tau} s_{\alpha}}{8 \sqrt{2} \pi c_{W}^{2} M_{1}}\left\{s_{2 \theta}^{2}\left[I_{3}\left(1, x_{2}, x_{2}\right)+I_{3}\left(1, x_{3}, x_{3}\right)\right]+2 c_{2 \theta}^{2} I_{3}\left(1, x_{2}, x_{3}\right)\right\} P_{R},
\end{aligned}
$$

with $c_{W}^{2} \equiv \cos ^{2} \theta_{W}, P_{R}=\left(1+\gamma_{5}\right) / 2, x_{i} \equiv \tilde{m}_{i}^{2} / M_{1}^{2}$, and

$$
I_{3}(x, y, z)=\frac{x y \log (x / y)+y z \log (y / z)+z x \log (z / x)}{(x-y)(y-z)(z-x)} .
$$

For brevity we suppressed in eq. (3.5) the spinors for the lepton fields. The amplitude for $h \rightarrow \tau^{-} \mu^{+}$is the same as the one in eq. (3.5) after exchanging $P_{R}$ with $P_{L}=\left(1-\gamma_{5}\right) / 2$.

The sum of the two amplitudes then reads:

$$
\begin{aligned}
& \mathcal{M}\left(h \rightarrow \tau^{+} \mu^{-}\right)=i \frac{\alpha A_{\mu \tau} s_{\alpha}}{8 \sqrt{2} \pi c_{W}^{2} M_{1}}\left\{s_{2 \theta}^{2}\right. {\left[I_{3}\left(1, x_{2}, x_{2}\right)+I_{3}\left(1, x_{3}, x_{3}\right)\right]+} \\
&\left.+2\left[c_{2 \theta}^{2}-s_{2 \theta} \frac{M_{1}^{2}\left(x_{2}-x_{3}\right)}{\sqrt{2} v c_{\beta} A_{\mu \tau}}\right] I_{3}\left(1, x_{2}, x_{3}\right)\right\} P_{R} .
\end{aligned}
$$

We see that in the limit of small mixing, $s_{2 \theta} \ll 1\left(v c_{\beta} A_{\mu \tau} \ll \tilde{m}_{2}^{2}-\tilde{m}_{3}^{2}\right)$, we have $\mathcal{M}(h \rightarrow \tau \mu)=\mathcal{O}\left(s_{2 \theta}^{2}\right)=\mathcal{O}\left(v^{2} / m_{\text {SUSY }}^{2}\right)$, as argued in the previous section. To estimate the largest possible value of $R_{\tau \mu / \tau \tau}$, we take, however, the limit of maximal mixing, $\sin ^{2} 2 \theta=1$. This limit is obtained by fine-tuning $\tilde{m}_{\mu_{L}}^{2}=\tilde{m}_{\tau_{R}}^{2}$ and, consequently, $\tilde{m}_{2}^{2}-\tilde{m}_{3}^{2}=\sqrt{2} v c_{\beta} A_{\mu \tau}$. In this case the sum of $\left|\mathcal{M}\left(h \rightarrow \tau^{+} \mu^{-}\right)\right|^{2}$ and $\left|\mathcal{M}\left(h \rightarrow \tau^{-} \mu^{+}\right)\right|^{2}$ reads

$$
|\mathcal{M}(h \rightarrow \tau \mu)|^{2}=m_{h}^{2} \frac{\alpha^{2} s_{\alpha}^{2}}{64 \pi^{2} c_{W}^{4}} \frac{A_{\mu \tau}^{2}}{M_{1}^{2}}\left[I_{3}\left(1, x_{2}, x_{2}\right)+I_{3}\left(1, x_{3}, x_{3}\right)-2 I_{3}\left(1, x_{2}, x_{3}\right)\right]^{2} .
$$

To check that our result has the correct decoupling behavior for $m_{\mathrm{SUSY}} \gg v$, we evaluate the terms in parenthesis in the limit $\left(\tilde{m}_{2}^{2}-\tilde{m}_{3}^{2}\right) \ll\left(\tilde{m}_{2}^{2}+\tilde{m}_{3}^{2}\right), M_{1}^{2}$ :

$$
|\mathcal{M}(h \rightarrow \tau \mu)|^{2}=m_{h}^{2} \frac{\alpha^{2} s_{\alpha}^{2}}{144 \pi^{2} c_{W}^{4}} \frac{A_{\mu \tau}^{2}}{M_{1}^{2}}\left(\frac{x_{2}-x_{3}}{x_{2}+x_{3}}\right)^{4} \frac{\left(1-6 x_{23}+3 x_{23}^{2}+2 x_{23}^{3}-6 x_{23}^{2} \log x_{23}\right)^{2}}{\left(1-x_{23}\right)^{8}},
$$


where $x_{23} \equiv\left(\tilde{m}_{2}^{2}+\tilde{m}_{3}^{2}\right) /\left(2 M_{1}^{2}\right)$. The amplitude indeed scales as $\frac{\left(x_{2}-x_{3}\right)^{2}}{\left(x_{2}+x_{3}\right)^{2}} \sim v^{2} / m_{\text {SUSY }}^{2}$.

The amplitude grows as $A_{\mu \tau}$. There is, however, a perturbativity bound on $A_{\mu \tau}$ :

$$
\left|\frac{A_{\mu \tau} s_{\alpha}}{\sqrt{2}}\right| \lesssim 4 \pi \tilde{m}_{3}
$$

The fine tuning in models with $m_{h} \ll A_{\mu \tau}$ is of order [30]

$$
\Delta \simeq \frac{1}{16 \pi^{2}} \frac{A_{\mu \tau}^{2}}{m_{h}^{2}}
$$

We denote the ratio $R_{\tau \mu / \tau \tau}$ that corresponds to maximal mixing, $s_{2 \theta}^{2}=1$, and maximal perturbative $A_{\mu \tau}=4 \sqrt{2} \pi \tilde{m}_{3} / s_{\alpha}$ by $R_{\tau \mu / \tau \tau}^{\max }$ :

$$
R_{\tau \mu / \tau \tau}^{\max }=\left\{\frac{\alpha}{2 c_{W}^{2}} \frac{v}{m_{\tau}} \frac{c_{\beta}}{s_{\alpha}} \frac{\tilde{m}_{3}}{M_{1}}\left[I_{3}\left(1, x_{2}, x_{2}\right)+I_{3}\left(1, x_{3}, x_{3}\right)-2 I_{3}\left(1, x_{2}, x_{3}\right)\right]\right\}^{2} .
$$

In figure 2 we show the value of $R_{\tau \mu / \tau \tau}^{\max }$ in the $\tilde{m}_{3}-M_{1}$ plane. Here, $A_{\mu \tau}=$ $4 \sqrt{2} \pi \tilde{m}_{3} /\left|s_{\alpha}\right|, \tilde{m}_{2}^{2}=\tilde{m}_{3}^{2}+\sqrt{2} v c_{\beta} A_{\mu \tau}$, and $\left|s_{\alpha} / c_{\beta}\right|=1$. We emphasize that $\left|s_{\alpha} / c_{\beta}\right|=0.75$ gives an $\mathcal{O}(1)$ enhancement but is forbidden by vacuum stability, which will be discussed in more detail below. Also depicted in this plot is the region excluded by the upper bound on $\tau \rightarrow \mu \gamma$ (for details see appendix C) and contours of deviation of the $h \rightarrow \gamma \gamma$ partial width with respect to the SM one (see appendix D).

We conclude that, with $A_{\mu \tau}$ being the source of LFV, we have

$$
R_{\tau \mu / \tau \tau} \lesssim 0.0015
$$

below the near-future sensitivity of ATLAS and CMS. Even by including both $A_{\mu \tau}$ and $A_{\tau \mu}$ at the same time, $R_{\tau \mu / \tau \tau} \lesssim 0.002$.

\section{$4 \quad$ LFV from the $\tilde{m}_{L}^{2}$ terms}

Consider the case that the sources of LFV are the matrices $\tilde{m}_{L}^{2}$ and $\tilde{m}_{R}^{2}$. To obtain $R_{\tau \mu / \tau \tau}$ as large as $\mathcal{O}(0.1)$, there must be no additional suppression from the mixing angle or from the loop function. At least one of $\tilde{m}_{L}^{2}$ and $\tilde{m}_{R}^{2}$ has to be anarchic in the $\mu-\tau$ sector to have a mixing angle of order one. While large $\tilde{\mu}-\tilde{\tau}$ mixing is a necessary condition, if it is large in both $\tilde{m}_{L}^{2}$ and $\tilde{m}_{R}^{2}$, the $\tau$-lepton in figure $1 \mathrm{~b}$ can be replaced with a muon, which implies that $\operatorname{BR}(h \rightarrow \mu \mu) \sim \operatorname{BR}(h \rightarrow \tau \mu)$. Given our requirement that $R_{\tau \mu / \tau \tau} \gtrsim 0.1$, and the experimental upper bound on $\operatorname{BR}(h \rightarrow \mu \mu)$ [31, 32], this case is disfavored. Thus, either $\tilde{m}_{L}^{2}$ or $\tilde{m}_{R}^{2}$ has to be near-diagonal. For concreteness, we take $\tilde{m}_{L}^{2}$ to be anarchic and $\tilde{m}_{R}^{2}$ to be diagonal. Hence, we focus on the $3 \times 3$ block of $\left(\tilde{\mu}_{L}, \tilde{\tau}_{L}, \tilde{\tau}_{R}\right)$ in the slepton mass-squared matrix.

The relevant part of the slepton mass-squared matrix has the form:

$$
\tilde{\mathcal{M}}^{2}=\left(\begin{array}{ccc}
\left(\tilde{m}_{L}^{2}\right)_{\mu \mu} & \left(\tilde{m}_{L}^{2}\right)_{\mu \tau} & 0 \\
\left(\tilde{m}_{L}^{2}\right)_{\mu \tau}^{*} & \left(\tilde{m}_{L}^{2}\right)_{\tau \tau} & -m_{\tau} \mu t_{\beta} \\
0 & -m_{\tau} \mu t_{\beta} & \left(\tilde{m}_{R}^{2}\right)_{\tau \tau}
\end{array}\right)
$$


where, for simplicity, we set $A^{E}=0$ and $y_{\mu}=0$.

We denote by $\tilde{U}$ the mixing matrix that rotates from the interaction basis $\left(\tilde{\mu}_{L}, \tilde{\tau}_{L}, \tilde{\tau}_{R}\right)$ to the mass basis $\left(\tilde{\ell}_{1}, \tilde{\ell}_{2}, \tilde{\ell}_{3}\right)$. To maximize the rate of $h \rightarrow \tau \mu$, it is best if the dominant contribution comes from the lightest slepton mass eigenstate, $\tilde{\ell}_{3}$. The mixing angles that enter the amplitude are

$$
\tilde{U}_{3 \mu_{L}}^{*} \tilde{U}_{3 \tau_{R}} \times 2 \mathcal{R} e\left(\tilde{U}_{3 \tau_{L}} \tilde{U}_{3 \tau_{R}}^{*}\right) .
$$

We are interested in estimating the largest possible contribution to $h \rightarrow \tau \mu$. Therefore, we are interested in the values of $\tilde{U}_{3 \alpha}$ that maximize eq. (4.1):

$$
\tilde{U}_{3 \alpha}=(1 / 2,1 / 2,1 / \sqrt{2}) .
$$

The way to achieve eq. (4.2) is by two tunings of entries of $\tilde{\mathcal{M}}^{2}$. First, we set $\left(\tilde{m}_{L}^{2}\right)_{\mu \mu}=$ $\left(\tilde{m}_{L}^{2}\right)_{\tau \tau}$. Then, we extract the two eigenvalues of the $\tilde{m}_{L}^{2}$ matrix. We take the heavier eigenvalue $\left(\tilde{m}_{L}^{2}\right)_{+}$to be very large, so that the corresponding mass eigenstate $\tilde{\ell}_{+}=\frac{1}{\sqrt{2}}\left(\tilde{\tau}_{L}+\tilde{\mu}_{L}\right)$ decouples. We are left with an effective two-slepton framework, $\tilde{\ell}_{-}=\frac{1}{\sqrt{2}}\left(\tilde{\tau}_{L}-\tilde{\mu}_{L}\right)$ and $\tilde{\tau}_{R}$ :

$$
\tilde{\mathcal{M}}^{2}=\left(\begin{array}{cc}
\left(\tilde{m}_{L}^{2}\right)_{-} & -m_{\tau} \mu t_{\beta} / \sqrt{2} \\
-m_{\tau} \mu t_{\beta} / \sqrt{2} & \left(\tilde{m}_{R}^{2}\right)_{\tau \tau}
\end{array}\right),
$$

where $\left(\tilde{m}_{L}^{2}\right)_{-}=\left(\tilde{m}_{L}^{2}\right)_{\tau \tau}-\left(\tilde{m}_{L}^{2}\right)_{\mu \tau}$. The mixing angle $\theta$, which rotates from the interaction basis $\left(\tilde{\ell}_{-}, \tilde{\tau}_{R}\right)$ to the mass basis $\left(\tilde{\ell}_{3}, \tilde{\ell}_{2}\right)$, is given by

$$
\tan 2 \theta=\frac{\sqrt{2} m_{\tau} \mu t_{\beta}}{\left(\tilde{m}_{L}^{2}\right)_{-}-\left(\tilde{m}_{R}^{2}\right)_{\tau \tau}} .
$$

The trilinear scalar couplings in the mass basis are given by

$$
\mathcal{L}_{h \tilde{\ell} \tilde{\ell}}=-\frac{m_{\tau} \mu c_{\alpha}}{\sqrt{2} v c_{\beta}} h\left[s_{2 \theta}\left(\tilde{\ell}_{2}^{*} \tilde{\ell}_{2}-\tilde{\ell}_{3}^{*} \tilde{\ell}_{3}\right)-c_{2 \theta}\left(\tilde{\ell}_{2}^{*} \tilde{\ell}_{3}+\tilde{\ell}_{3}^{*} \tilde{\ell}_{2}\right)\right],
$$

and the bino-lepton-slepton couplings are given by

$$
\mathcal{L}_{\tilde{B} \tilde{\ell} \ell}=\frac{g^{\prime}}{2} \tilde{B}\left(c_{\theta} \tilde{\ell}_{2}^{*}+s_{\theta} \tilde{\ell}_{3}^{*}\right)\left(\tau_{L}-\mu_{L}\right)+\sqrt{2} g^{\prime} \tilde{B}\left(s_{\theta} \tilde{\ell}_{2}-c_{\theta} \tilde{\ell}_{3}\right) \bar{\tau}_{R}+\text { h.c. }
$$

Given this Lagrangian we can compute the $h \rightarrow \tau \mu$ amplitude along lines similar to the analysis of the LFV $A$-terms. There is the one-loop contribution (figure 1b) and field renormalization contribution (figure 1c).

As a second step in obtaining optimal mixing, we tune $\left(\tilde{m}_{L}^{2}\right)_{-}=\left(\tilde{m}_{R}^{2}\right)_{\tau \tau}$ to generate maximal $\tilde{\ell}_{-}-\tilde{\tau}_{R}$ mixing. This tunning fixes the mass difference of the two slepton mass eigenstates to be $\tilde{m}_{2}^{2}-\tilde{m}_{3}^{2}=\sqrt{2} m_{\tau} \mu t_{\beta}$. We obtain, for the specific mixing pattern of eq. (4.2):

$$
\begin{aligned}
\mathcal{M}\left(h \rightarrow \tau^{+} \mu^{-}\right)=-i & \frac{\alpha \mu m_{\tau}}{16 \pi c_{W}^{2} M_{1} v}\left\{c_{\beta-\alpha}\left[I_{3}\left(1, x_{2}, x_{2}\right)+I_{3}\left(1, x_{3}, x_{3}\right)+2 t_{\beta}^{2} I_{3}\left(1, x_{2}, x_{3}\right)\right]\right. \\
& \left.+s_{\beta-\alpha} t_{\beta}\left[I_{3}\left(1, x_{2}, x_{2}\right)+I_{3}\left(1, x_{3}, x_{3}\right)-2 I_{3}\left(1, x_{2}, x_{3}\right)\right]\right\} P_{R} .
\end{aligned}
$$


We wrote this expression in a way that transparently exposes the correct decoupling limit. We remind the reader that both $m_{\tau} / v$ and $\mu / M_{1}$ are finite in the decoupling limit. Then, the first term scales like $c_{\beta-\alpha}$ and the second term scales like $\left(\tilde{m}_{2}^{2}-\tilde{m}_{3}^{2}\right)^{2} /\left(\tilde{m}_{2}^{2}+\tilde{m}_{3}^{2}\right)^{2}$, both of which are proportional to $v^{2} / m_{\text {SUSY }}^{2}$.

For the ratio of branching ratios, we find:

$$
R_{\tau \mu / \tau \tau}=\left(\frac{\alpha \mu}{16 \pi c_{W}^{2} M_{1}}\right)^{2}\left[2 t_{\beta} I_{3}\left(1, x_{2}, x_{3}\right)-\frac{c_{\beta-\alpha}+s_{\beta-\alpha} t_{\beta}}{s_{\beta-\alpha}-c_{\beta-\alpha} t_{\beta}} \sum_{i=2,3} I_{3}\left(1, x_{i}, x_{i}\right)\right]^{2} .
$$

The ratio grows as $\left(\mu / M_{1}\right)^{2}$. However, there is a perturbativity bound on $\mu$ :

$$
\frac{m_{\tau} \mu\left(c_{\beta-\alpha}+s_{\beta-\alpha} t_{\beta}\right)}{\sqrt{2} v} \lesssim 4 \pi \tilde{m}_{3} .
$$

The fine tuning in models with $m_{h} \ll \mu$ is of order [30]

$$
\Delta \simeq \frac{2 \mu^{2}}{m_{h}^{2}} .
$$

We denote the ratio $R_{\tau \mu / \tau \tau}$ which corresponds to $\tilde{U}_{3 \alpha}=(1 / 2,1 / 2,1 / \sqrt{2})$ and to $\mu$ at the perturbative bound of eq. (4.8) by $R_{\tau \mu / \tau \tau}^{\max }$ :

$$
R_{\tau \mu / \tau \tau}^{\max }=\left\{\frac{\alpha v \sqrt{x_{3}}}{2 \sqrt{2} m_{\tau} c_{W}^{2}}\left[\frac{2 I_{3}\left(1, x_{2}, x_{3}\right)}{s_{\beta-\alpha}+c_{\beta-\alpha} / t_{\beta}}-\frac{\sum_{i=2,3} I_{3}\left(1, x_{i}, x_{i}\right)}{s_{\beta-\alpha}-c_{\beta-\alpha} t_{\beta}}\right]\right\}^{2} .
$$

The parameters $c_{\beta-\alpha}$ and $t_{\beta}$ play a crucial role on the value of $R_{\tau \mu / \tau \tau}^{\max }$. The allowed range in the $c_{\beta-\alpha}-t_{\beta}$ plane is shown in figure 6 of appendix $\mathrm{B}$. The upper bound on $R_{\tau \mu / \tau \tau}^{\max }$ is different for the bulk region and the peninsula region. The peninsula region corresponds to the parameter space in which the $h V V$ and $h \gamma \gamma$ couplings are close to their SM values, while the $h \tau \tau$ coupling has the same absolute value but opposite sign.

It is interesting to note that, for $M_{1}, \tilde{m}_{3} \gg v$, the sleptons are quasi-degenerate and eq. (4.10) takes the form

$$
R_{\tau \mu / \tau \tau}^{\max }=\left\{\frac{\alpha v}{\sqrt{2} m_{\tau} c_{W}^{2}} \sqrt{x_{3}} I_{3}\left(1, x_{3}, x_{3}\right)\left[\frac{c_{\beta-\alpha} t_{\beta}}{s_{\beta-\alpha}\left(s_{\beta-\alpha}-c_{\beta-\alpha} t_{\beta}\right)}\right]\right\}^{2} .
$$

The maximum of the loop function is for $\tilde{m}_{3} / M_{1} \approx 0.47$ independently of their individual values. The best fit point for the trigonometric factor depends on whether we are in the bulk or in the peninsula regions.

In the right panels of figures 3 and 4 we show the value of $R_{\tau \mu / \tau \tau}^{\max }$ in the $\tilde{m}_{3}-M_{1}$ plane. Here, $m_{\tau} \mu=4 \sqrt{2} \pi \tilde{m}_{3} v /\left(c_{\beta-\alpha}+s_{\beta-\alpha} t_{\beta}\right)$, and $\tilde{m}_{2}^{2}=\tilde{m}_{3}^{2}+\sqrt{2} m_{\tau} \mu t_{\beta}$. Also depicted in these plots is the region excluded by the upper bound on $\tau \rightarrow \mu \gamma$ (see appendix C) and the deviation of the partial width of $h \rightarrow \gamma \gamma$ with respect to the SM (see appendix D).

Figure 3 corresponds to $c_{\beta-\alpha}$ and $t_{\beta}$ in the bulk region. We conclude that, with $\left(\tilde{m}_{L}^{2}\right)_{\mu \tau}$ being the source of LFV while also being in the bulk region

$$
R_{\tau \mu / \tau \tau} \lesssim 0.035 \quad \text { for }\left|c_{\beta-\alpha} t_{\beta}\right| \ll 1,
$$



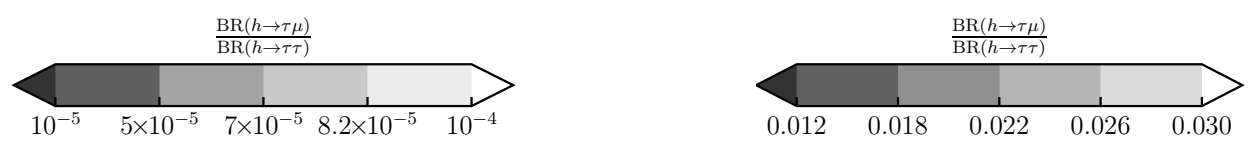

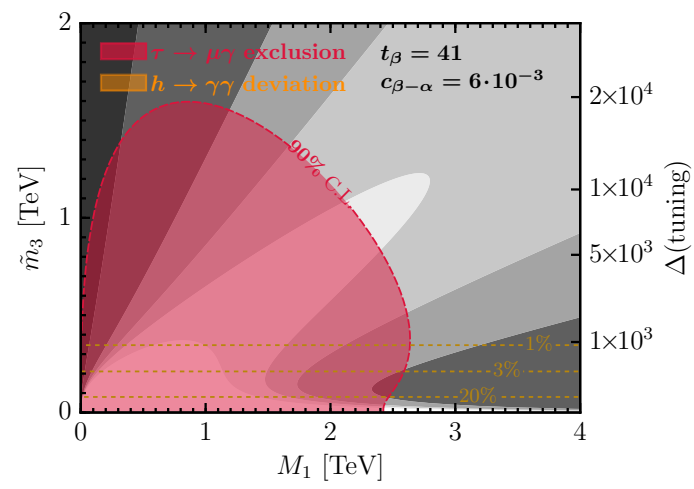

(a)

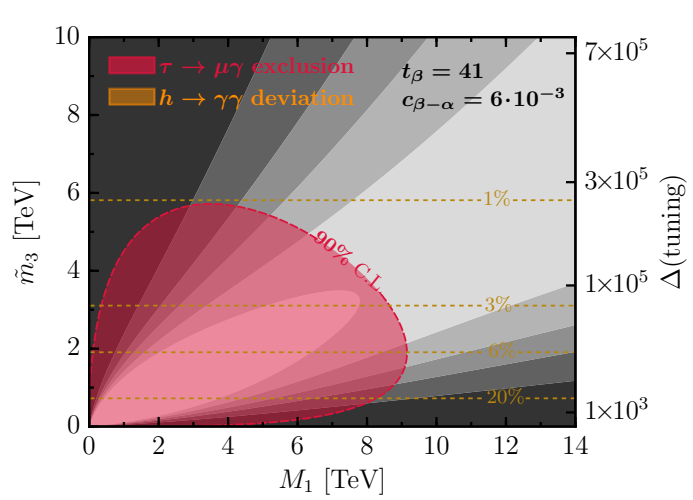

(b)

Figure 3. Contours of $R_{\tau \mu / \tau \tau}$ in the $M_{1}-\tilde{m}_{3}$ for the case of LFV from the slepton mass-squared term $\left(\tilde{m}_{L}^{2}\right)_{\mu \tau}$. For $\cos (\beta-\alpha)$ and $\tan \beta$ we take the values in the bulk region of figure 6 that maximize $R_{\tau \mu / \tau \tau}$. In the left (right) panel the value of the $\mu$ is the maximal allowed by vacuum stability (perturbativity); the associated tuning is indicated on the right y-axis. The red region in dashed lines is excluded by the bound on $\tau \rightarrow \mu \gamma$. Orange horizontal lines indicate the deviation of the partial $h \rightarrow \gamma \gamma$ width with respect to the SM value.

below the near-future sensitivity of ATLAS and CMS.

Figure 4 corresponds to $c_{\beta-\alpha}$ and $t_{\beta}$ in the peninsula region. Here, much higher values of $R_{\tau \mu / \tau \tau}$ can be reached. In particular, the present upper bound on this ratio (eq. (1.4)),

$$
R_{\tau \mu / \tau \tau} \lesssim 0.31 \quad \text { for } c_{\beta-\alpha} t_{\beta} \simeq 2 \text {, }
$$

can be saturated.

\section{Charge breaking minima}

In previous sections we established that large trilinear scalar couplings enable, in principle, enhancement of the MSSM contributions to $h \rightarrow \tau \mu$ well above the estimate from naive dimensional analysis. Such large trilinear couplings might lead, however, to charge breaking minima that are lower than the electroweak symmetry breaking one. In this section we obtain upper bounds on the trilinear couplings by requiring that the global minimum is not charge breaking.

We are particularly concerned whether the corner of parameter space, in which the MSSM saturates the upper bound (see eq. (4.13)) while being consistent with experimental constraints and the perturbativity bound, does not lead to a global minimum that is charge breaking. The relevant scalar fields are the Higgs and slepton fields. Similar bounds on $\mu$ have been investigated in the past [33-37]. In our case, the dangerous directions in the field 

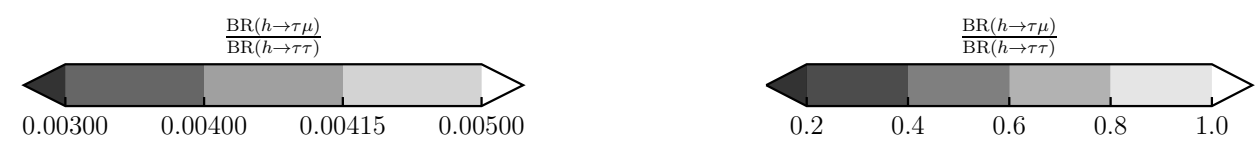

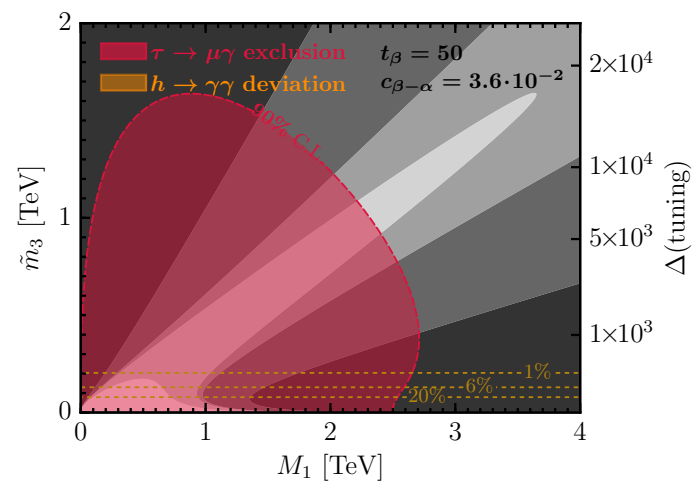

(a)

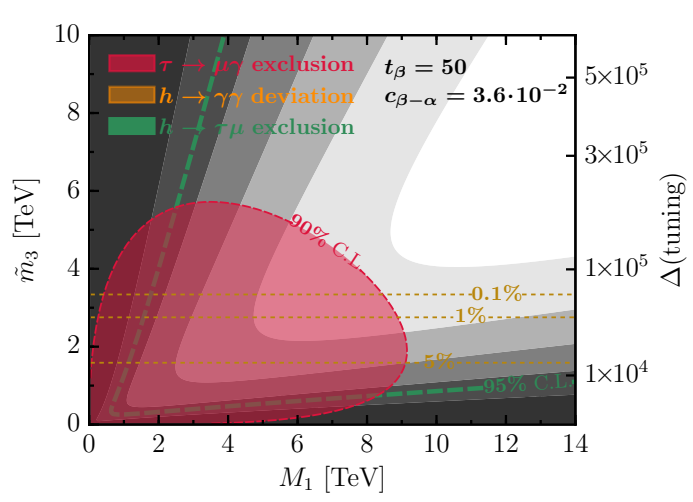

(b)

Figure 4. Contours of $R_{\tau \mu / \tau \tau}$ in the $M_{1}-\tilde{m}_{3}$ for the case of LFV from the slepton mass-squared term $\left(\tilde{m}_{L}^{2}\right)_{\mu \tau}$. For $\cos (\beta-\alpha)$ and $\tan \beta$ we take the values in the peninsula region of figure 6 that maximize $R_{\tau \mu / \tau \tau}$. In the left (right) panel the value of the $\mu$ is the maximal allowed by vacuum stability (perturbativity); the associated tuning is indicated on the right y-axis. The red region in dashed lines is excluded by the bound on $\tau \rightarrow \mu \gamma$. Orange horizontal lines indicate the deviation of the partial $h \rightarrow \gamma \gamma$ width with respect to the SM value. The green dashed line indicates the direct upper bound on $R_{\tau \mu / \tau \tau}$.

space are those where there is no dependence on the heavy slepton mass-squared eigenvalue $\tilde{m}_{L_{+}}^{2}$. Thus, we focus on the following direction:

$$
\left\langle H_{u}^{0}\right\rangle=\left\langle\tilde{\tau}_{R}\right\rangle=\left\langle\tilde{\ell}_{-}\right\rangle=f .
$$

In this direction, the soft breaking terms and F-terms are the following:

$$
\begin{aligned}
V_{F+\text { soft }}= & \tilde{m}_{L_{-}}^{2}\left\langle\tilde{\ell}_{-}^{*}\right\rangle\left\langle\tilde{\ell}_{-}\right\rangle+\tilde{m}_{R}^{2}\left\langle\tilde{\tau}_{R}^{*}\right\rangle\left\langle\tilde{\tau}_{R}\right\rangle+\frac{1}{\sqrt{2}} y_{\tau} \mu\left(\left\langle H_{u}\right\rangle\left\langle\tilde{\tau}_{R}^{*}\right\rangle\left\langle\tilde{\ell}_{-}\right\rangle+\text {h.c. }\right) \\
& +\frac{1}{2} y_{\tau}^{2}\left\langle\tilde{\tau}_{R}^{*}\right\rangle\left\langle\tilde{\tau}_{R}\right\rangle\left\langle\tilde{\ell}_{-}^{*}\right\rangle\left\langle\tilde{\ell}_{-}\right\rangle
\end{aligned}
$$

where we have used that, for $t_{\beta} \gg 1$ and $c_{\beta-\alpha} \ll 1,\left(M_{H_{u}}^{2}+|\mu|^{2}\right) \ll \Lambda_{\text {SUSY }}^{2}$ to neglect the contribution from the $\left\langle H_{u}\right\rangle^{2}$ term. Using our ansatz, $\tilde{m}_{L_{-}}^{2}=\tilde{m}_{R}^{2}=\frac{1}{2}\left(\tilde{m}_{2}^{2}+\tilde{m}_{3}^{2}\right)$, and adding the D-term, we obtain

$$
V \simeq\left(\tilde{m}_{2}^{2}+\tilde{m}_{3}^{2}+\sqrt{2} y_{\tau} \mu f+\frac{y_{\tau}^{2}+g^{2}+g^{\prime 2}}{2} f^{2}\right) f^{2} .
$$

We require that the minimum at $f=0$ should be deeper than minima with $f \neq 0$. This is equivalent to requiring that the discriminant for the term in parenthesis is negative:

$$
\mu^{2} \leq\left(\tilde{m}_{2}^{2}+\tilde{m}_{3}^{2}\right) \frac{y_{\tau}^{2}+g^{2}+g^{\prime 2}}{y_{\tau}^{2}} .
$$


Using the relation that follows from our ansatz, $\tilde{m}_{2}^{2}=\tilde{m}_{3}^{2}+\sqrt{2} m_{\tau} \mu t_{\beta}$, we find, to leading order in $v / \tilde{m}_{3}$,

$$
|\mu| \lesssim \sqrt{\frac{2}{y_{\tau}^{2}}\left(y_{\tau}^{2}+g^{2}+g^{\prime 2}\right)} \tilde{m}_{3} \sim 2.5 \tilde{m}_{3},
$$

where in the last equation we use $y_{\tau} \sim 0.5$ (corresponding to $t_{\beta} \sim 50$ ). For $\tilde{m}_{3}$ as low as $0.5 \mathrm{TeV}$, the bound is relaxed to $|\mu| \lesssim 2.9 \tilde{m}_{3}$. In any case, the bound from charge breaking minimum is much stronger than the bound from perturbativity, eq. (4.8). In the left panels of figures 3 and 4 we show the value of $R_{\tau \mu / \tau \tau}^{\max }$ in the $\tilde{m}_{3}-M_{1}$ plane. Here we use the maximal value of $\mu$ allowed by the charged breaking constraint.

When we replace the perturbativity bound with the one from charge breaking minima, we obtain, in the bulk region,

$$
R_{\tau \mu / \tau \tau} \lesssim 10^{-4} \quad \text { for }\left|c_{\beta-\alpha} t_{\beta}\right| \ll 1,
$$

to be compared with eq. (4.12), and in the peninsula region,

$$
R_{\tau \mu / \tau \tau} \lesssim 4 \times 10^{-3} \quad \text { for } c_{\beta-\alpha} t_{\beta} \simeq 2,
$$

to be compared with eq. (4.13).

The bound on $|\mu|$ becomes weaker if, instead of requiring the absence of charge breaking minima, we would only require that the electroweak breaking minimum is metastable with a lifetime that is longer than the age of the Universe. Following the analysis of ref. [34] we find that the bound on $|\mu|$ is relaxed by at most a factor of two compared to the one from eq. (5.5). (When $\mu$ saturates the perturbative bound of eq. (4.8) the lifetime of the electroweak minimum is exponentially smaller than the age of the Universe.) Therefore, our conclusions remain unchanged.

The case of the $A$-term is somewhat different. The analysis proceeds along similar lines. One can avoid, however, the existence of deeper minima by taking the soft SUSY breaking parameter $M_{H_{d}}$ to be much larger than the $A$-term itself (which is equivalent to the limit $c_{\beta-\alpha} \rightarrow 0$ ). In this case the final result changes only by a factor of 2 .

We conclude that while the MSSM can enhance $R_{\tau \mu / \tau \tau}$ by some three orders of magnitude compared to the naive estimate of $(\alpha / 4 \pi)^{2}$, its maximal contribution is still about two orders of magnitude below the near future experimental sensitivity. It is interesting to note that an enhancement of the same order of magnitude can be achieved if all possible MSSM contributions to the $h \rightarrow \tau \mu$ decay interfere constructively, even if the trilinear scalar couplings do not saturate their upper bounds. In either case, such an enhancement arises only in non-generic regions of the parameter space.

\section{Conclusions}

The ATLAS and CMS experiments can discover the lepton-flavor violating Higgs decay $h \rightarrow \tau \mu$ if its rate is not much lower than the rate of the $h \rightarrow \tau \tau$ decay. We examined the question of whether the minimal supersymmetric Standard Model (MSSM) will be unambiguously excluded in case such a discovery is made. The version of the MSSM that we analyzed has the following features: 
- R-parity is conserved.

- Non-renormalizable terms are negligible.

- All couplings are perturbative. They need not obey, however, any other principle, such as flavor universality.

- There is no charge breaking minimum that is deeper than the electroweak symmetry breaking one.

Since in this framework the $h \rightarrow \tau \mu$ decay is suppressed by an electroweak loop, while the $h \rightarrow \tau \tau$ decay proceeds at tree level, in generic points of the MSSM parameter space the LFV decay is suppressed to values orders of magnitude below the sensitivity of the LHC experiments.

When we consider only the perturbativity bounds on trilinear scalar couplings, we find very non-generic points in the MSSM parameter space that can compensate for the electroweak loop suppression. Specifically, if $\operatorname{BR}(h \rightarrow \tau \mu) / \operatorname{BR}(h \rightarrow \tau \tau)$ is discovered with a value close to the present experimental bound, the MSSM with perturbative couplings can account for it under the following, highly non-generic, conditions: (i) The $\mu$-term is close to its perturbative bound; (ii) There is order one lepton-flavor violation (in the $\tau-\mu$ sector) in one of the slepton mass-squared matrices, and very small lepton-flavor violation in the other; (iii) The bino and the sleptons have masses at the $\mathrm{TeV}$ scale or higher, and the higgsinos are an order of magnitude heavier; (iv) The two lightest sleptons are quasi-degenerate; (v) The second Higgs doublet is lighter than the sleptons and bino.

The MSSM at this corner of parameter space has, however, a charge breaking minimum that is lower than the electroweak symmetry breaking minimum. Avoiding such a minimum (or even just requiring that the lifetime of the electroweak minimum is longer than the age of the Universe) is incompatible with the condition (i). Thus, the $\mu$-parameter has to be smaller than the perturbative bound by about an order of magnitude, suppressing the ratio $R_{\tau \mu / \tau \tau} \equiv \mathrm{BR}(h \rightarrow \tau \mu) / \mathrm{BR}(h \rightarrow \tau \tau)$ by at least two orders of magnitude compared to the present experimental sensitivity.

We conclude that if ATLAS and CMS establish that $R_{\tau \mu / \tau \tau} \gtrsim 10^{-2}$, the R-parity conserving MSSM will be excluded.

Note added. While this work was in writing, ref. [38] appeared which also examines the possibility of a large rate for $h \rightarrow \tau \mu$ within the MSSM. As far as a comparison is possible, we agree with their results. In particular, the importance of the constraints from $\tau \rightarrow \mu \gamma$ is emphasized. However, ref. [38] calculates $\mathrm{BR}(h \rightarrow \tau \mu)$ and not the ratio $R_{\tau \mu / \tau \tau}$. Thus, the entire MSSM spectrum and mixing needs to be specified, which dictates the Higgs total width as well as the spectrum and couplings of the $H^{0}, A^{0}$ and $H^{ \pm}$scalar particles. With their specific choice, much smaller rates for $h \rightarrow \tau \mu$ are obtained. In the present work, on the other hand, we are mainly interested in the possibility to unambiguously exclude the MSSM. We thus allow for the possibility that the squark sector strongly affects the total width and the Higgs potential, so that generic constraints related to these aspects cannot be applied. 


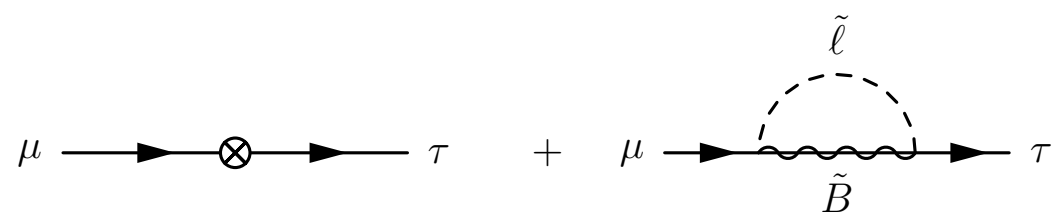

Figure 5. Counterterm and one-loop contribution to the flavor off-diagonal $\mu \rightarrow \tau$ two-point function.

\section{Acknowledgments}

We thank Kfir Blum, Avital Dery, Aielet Efrati, Claudia Frugiuele and Yotam Soreq for valuable discussions. We are grateful to Roni Harnik for pointing out the potential importance of charge breaking minima to our analysis. YN is the Amos de-Shalit chair of theoretical physics. YN is supported by the I-CORE program of the Planning and Budgeting Committee and the Israel Science Foundation (grant number 1937/12), and by a grant from the United States-Israel Binational Science Foundation (BSF), Jerusalem, Israel.

\section{A Field renormalization}

To compute the transition amplitudes of physical particles, like the $h \rightarrow \tau \mu$ transition presented in sections 3 and 4 , it is convenient to work with a Lagrangian in which all external fields have canonically normalized kinetic terms. Since at the loop level this, a priori, does not hold, it is a convenient and standard procedure to include finite parts in all the field renormalization constants of external particles to enforce proper kinetic terms also at the loop-level. This makes it manifest that the species of an on-shell particle cannot change in the vacuum and is the way field renormalization constants are implemented in the on-shell scheme.

Below we outline the procedure in the example of the $h \rightarrow \tau \mu$ decay. In terms of Dirac fields, the relevant Lagrangian terms read:

$$
\mathcal{L}_{\tau \tau}=\bar{\tau}^{0} i \not D \tau^{0}-\left(m_{\tau}^{0}+\frac{m_{\tau}^{0}}{v^{0}} \frac{\sin \alpha^{0}}{\cos \beta^{0}} h^{0}\right)\left(\bar{\tau}_{L}^{0} \tau_{R}^{0}+\bar{\tau}_{R}^{0} \tau_{L}^{0}\right)
$$

where the superscript " 0 " indicates bare fields and parameters. In a chiral field theory like the MSSM the fields of different chirality are renormalized individually. The lepton fields are renormalized via the field renormalization constants

$$
\ell_{A_{i}}^{0}=\left(Z_{i j}^{A}\right)^{1 / 2} \ell_{A_{j}} \equiv\left(\delta_{i j}+\frac{1}{2} \delta Z_{i j}^{A}+\mathcal{O} \text { (higher orders) }\right) \ell_{A_{j}} \quad \text { with } \quad A=L, R .
$$

Since we are only interested in tree-level counterterms mediating LFV transitions, the only relevant renormalization constants at one-loop are the above off-diagonal field renormalizations. By inserting the expansion in eq. (A.2) into eq. (A.1), we obtain the counterterms involving both $\tau$ and $\mu$ fields. We are interested in the off-diagonal terms, which are, in the limit of a massless muon,

$$
\mathcal{L}_{\mu \tau}=\frac{1}{2}\left(\delta Z_{\tau \mu}^{L}+\delta Z_{\mu \tau}^{L *}\right) \bar{\tau}_{L} i \not D \mu_{L}+\frac{1}{2}\left(\delta Z_{\tau \mu}^{R}+\delta Z_{\mu \tau}^{R *}\right) \bar{\tau}_{R} i \not \mu_{R}
$$




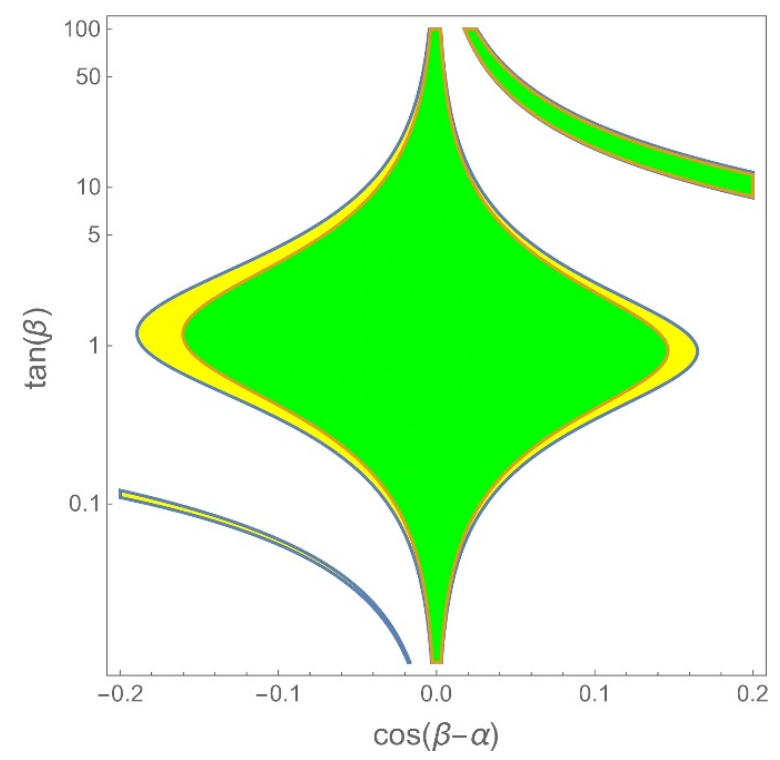

Figure 6. Allowed region in the $\cos (\beta-\alpha)-\tan \beta$ plane.

$$
-\left(m_{\tau}+\frac{m_{\tau}}{v} \frac{\sin \alpha}{\cos \beta} h\right) \frac{1}{2} \delta Z_{\tau \mu}^{R} \bar{\tau}_{L} \mu_{R}-\left(m_{\tau}+\frac{m_{\tau}}{v} \frac{\sin \alpha}{\cos \beta} h\right) \frac{1}{2} \delta Z_{\tau \mu}^{L} \bar{\tau}_{R} \mu_{L}+\text { h.c. . }
$$

We see that the $h-\tau-\mu$ vertex is renormalised by the same two constants, $\delta Z_{\tau \mu}^{L}$ and $\delta Z_{\tau \mu}^{R}$ as the "mass" vertex. The requirement that the off-diagonal two-point vanishes at all orders in perturbation theory fixes the two relevant constants, i.e. the sum of the diagrams in figure 5 should vanish. In our case it is sufficient to include only the part of the two-point function proportional to $m_{\tau}$ because the expansion in external momenta over SUSY masses is a good approximation:

$$
\begin{aligned}
0 \stackrel{!}{=} \mathcal{M}(\mu \rightarrow \tau) \supset & -i \frac{m_{\tau}}{2}\left(\delta Z_{\tau \mu}^{R} P_{R}+\delta Z_{\tau \mu}^{L} P_{L}\right) \\
& +i \sum_{i=2,3} \int \frac{d^{d} q}{(2 \pi)^{d}} \frac{\left(g_{L}^{\tau, i} P_{R}+g_{R}^{\tau, i} P_{L}\right)\left(\not q+M_{1}\right)\left(g_{L}^{\mu, i} P_{L}+g_{R}^{\mu, i} P_{R}\right)}{\left(q^{2}-M_{1}^{2}\right)\left(q^{2}-\tilde{m}_{i}^{2}\right)}
\end{aligned}
$$

where $g_{L, R}^{\alpha, i}$ denote the couplings between $\ell_{\alpha}-\tilde{\ell}_{i}-\tilde{B}$. In the case of our effective two-slepton framework, we find:

$$
\begin{aligned}
& \delta Z_{\tau \mu}^{R}=0, \\
& \delta Z_{\tau \mu}^{L}=-\frac{e^{2} s_{2 \theta}}{16 \pi^{2} c_{W}^{2}} \frac{\tilde{m}_{2}^{2}-\tilde{m}_{3}^{2}}{m_{\tau} M_{1}} I_{3}\left(1, x_{2}, x_{3}\right) \times\left\{\begin{array}{cc}
1 & \left(\text { from } A^{E}\right) \\
\frac{1}{\sqrt{2}} & \left(\text { from } \mu Y^{E}\right)
\end{array}\right.
\end{aligned}
$$

where $x_{i}=\tilde{m}_{i}^{2} / M_{1}^{2}$.

\section{B Constraints in the $\cos (\beta-\alpha)-\tan \beta$ plane}

The couplings of the light Higgs $h$ to $V V, \gamma \gamma, \tau \tau$ and $\tau \mu$ depend on $\tan \beta$ and $\cos (\beta-\alpha)$. We use the experimental constraints on the $h \rightarrow V V, \gamma \gamma$ and $\tau \tau$ modes to find the allowed 
region in the $\cos (\beta-\alpha)-\tan \beta$ plane. We do not use the data on $h \rightarrow b \bar{b}$, as we do not commit to a specific squark sector. We use the same set of data and procedure as employed in ref. [39]. To do so, we fix $\tan \beta$ and $\cos (\beta-\alpha)$ to all orders in perturbation theory through:

$$
\begin{aligned}
g_{h V V} / g_{h V V}^{\mathrm{SM}} & \equiv \sin (\beta-\alpha), \\
g_{h \tau \tau} / g_{h \tau \tau}^{\mathrm{SM}} & \equiv \sin (\beta-\alpha)-\cos (\beta-\alpha) \tan \beta .
\end{aligned}
$$

Note that this definition of $\tan \beta$ differs at the loop level from the standard definition of $v_{u} / v_{d}$. With this definition, our results should reproduce, to a good approximation, the allowed region for $2 \mathrm{HDM}$ Type II obtained in ref. [39], and indeed they do.

The allowed region in the $\cos (\beta-\alpha)-\tan \beta$ is presented in figure 6 . We refer to the central allowed region, which includes the point $(0,1)$ as "the bulk region". We call the branch in the upper right corner "the peninsula region", a term used in ref. [39]. It corresponds to $\sin (\beta-\alpha)$ not far from 1 , and $\cos (\beta-\alpha) \tan \beta$ not far from 2 . In this way, the $h V V$ and $h \gamma \gamma$ couplings are close to their SM values, while the $h \tau \tau$ coupling has the same absolute value but opposite sign.

\section{C $\tau \rightarrow \mu \gamma$}

To obtain the rate for $\tau \rightarrow \mu \gamma$, we integrate out all the heavy degrees of freedom, and match to the effective Lagrangian

$$
\mathcal{L}_{\text {eff }}=-\frac{e}{2} C_{\gamma} \bar{\mu}_{L} \sigma^{\mu \nu} \tau_{R} F_{\mu \nu}+\text { h.c. }
$$

The Wilson coefficient $C_{\gamma}$ gives the rate:

$$
\frac{\operatorname{BR}(\tau \rightarrow \mu \gamma)}{\operatorname{BR}(\tau \rightarrow \mu \nu \bar{\nu})}=\frac{48 \pi^{3} \alpha}{G_{F}^{2} m_{\tau}^{2}}\left|C_{\gamma}\right|^{2} .
$$

The relevant experimental results are $[40,41]$

$$
\begin{aligned}
\operatorname{BR}(\tau \rightarrow \mu \gamma) & <4.5 \times 10^{-8} \text { at } 90 \% \text { C.L. }, \\
\operatorname{BR}(\tau \rightarrow \mu \nu \bar{\nu}) & =(17.41 \pm 0.04) \times 10^{-2} .
\end{aligned}
$$

With this experimental input the bound on the Wilson coefficient reads:

$$
\left|C_{\gamma}\right|<3.1 \times 10^{-9} \mathrm{GeV}^{-1}
$$

In the cases of interest to us, we have an effective two-slepton framework, with the mass eigenstates $\left(\tilde{\ell}_{2}, \tilde{\ell}_{3}\right)$. We denote the mixing matrix for bino-slepton-lepton couplings by $\tilde{U}$. We obtain:

$$
C_{\gamma}=-\frac{\alpha}{8 \pi c_{W}^{2} M_{1}} \sum_{i=2,3} \tilde{U}_{i \mu_{L}} \tilde{U}_{i \tau_{R}}^{*} \frac{1-x_{i}^{2}+2 x_{i} \log x_{i}}{\left(1-x_{i}\right)^{3}}
$$


where $x_{i} \equiv \tilde{m}_{i}^{2} / M_{1}^{2}$. Then, the upper bound on $\left|C_{\gamma}\right|$ (C.4) translates into

$$
\frac{130 \mathrm{TeV}}{M_{1}}\left|\sum_{i=2,3} \tilde{U}_{i \mu_{L}} \tilde{U}_{i \tau_{R}}^{*} \frac{1-x_{i}^{2}+2 x_{i} \log x_{i}}{\left(1-x_{i}\right)^{3}}\right|<1
$$

For the case of LFV from the $A$-terms discussed in section 3 , we have $\tilde{U}_{2 \mu_{L}} \tilde{U}_{2 \tau_{R}}^{*}=$ $-\tilde{U}_{3 \mu_{L}} \tilde{U}_{3 \tau_{R}}^{*}=1 / 2$. For the case of LFV from the $\tilde{m}_{L}^{2}$-terms discussed in section 4 , we have $\tilde{U}_{2 \mu_{L}} \tilde{U}_{2 \tau_{R}}^{*}=-\tilde{U}_{3 \mu_{L}} \tilde{U}_{3 \tau_{R}}^{*}=1 /(2 \sqrt{2})$. Thus the contribution to $\left|C_{\gamma}\right|^{2}$ is smaller by a factor of $1 / 2$ in the latter case, compared to the former, and the lower bounds on the spartner masses from $\tau \rightarrow \mu \gamma$ are correspondingly weaker. In either case, taking into account that the large slepton mixing entails quasi-degeneracy between the two sleptons, we expect the lower bound on the bino and/or slepton masses to be of order $10 \mathrm{TeV}$. The numerical impact is shown in the relevant sections.

\section{$\mathrm{D} \quad h \rightarrow \gamma \gamma$}

Within the MSSM, the $h \rightarrow \gamma \gamma$ decay rate is given by

$$
\Gamma(h \rightarrow \gamma \gamma)=\frac{G_{F}^{2} \alpha^{2} m_{h}^{3}}{128 \sqrt{2} \pi^{3}}\left|\sum_{f} c_{f} A_{1 / 2}\left(\tau_{f}\right)+c_{w} A_{1}\left(\tau_{W}\right)+A_{\tilde{\ell}}\right|^{2},
$$

where $\tau_{f, W} \equiv m_{h}^{2} / 4 m_{f, W}^{2}$,

$$
\begin{aligned}
A_{1 / 2}(\tau) & =2[\tau+(\tau-1) f(\tau)] / \tau^{2} \\
A_{1}(\tau) & =-\left[2 \tau^{2}+3 \tau+3(2 \tau-1) f(\tau)\right] / \tau^{2},
\end{aligned}
$$

and

$$
f(\tau)=\left\{\begin{array}{cr}
\arcsin ^{2} \sqrt{\tau} & \tau \leq 1, \\
-\frac{1}{4}\left[\log \frac{1+\sqrt{1-\tau^{-1}}}{1-\sqrt{1-\tau^{-1}}}-i \pi\right]^{2} & \tau>1 .
\end{array}\right.
$$

The first term in eq. (D.1) comes from the SM fermion loops and the second from the $W$-boson loop, but with the MSSM couplings:

$$
\begin{aligned}
c_{t} & =\frac{4}{3}\left(s_{\beta-\alpha}+c_{\beta-\alpha} / t_{\beta}\right), \\
c_{b} & =\frac{1}{3}\left(s_{\beta-\alpha}-c_{\beta-\alpha} t_{\beta}\right), \\
c_{\tau} & =s_{\beta-\alpha}-c_{\beta-\alpha} t_{\beta}, \\
c_{w} & =s_{\beta-\alpha} .
\end{aligned}
$$

The third term comes from the slepton loop and, for $\tilde{m}_{2,3} \gg v$, is given by

$$
A_{\tilde{\ell}}=\frac{1}{6 \sqrt{2}}\left(\frac{1}{\tilde{m}_{2}^{2}}-\frac{1}{\tilde{m}_{3}^{3}}\right) \times\left\{\begin{array}{cc}
-s_{\alpha} v A_{\mu \tau} & \left(\text { from } A^{E}\right), \\
\left(c_{\beta-\alpha}+s_{\beta-\alpha} t_{\beta}\right) m_{\tau} \mu & \left(\text { from } \mu Y^{E}\right) .
\end{array}\right.
$$


Open Access. This article is distributed under the terms of the Creative Commons Attribution License (CC-BY 4.0), which permits any use, distribution and reproduction in any medium, provided the original author(s) and source are credited.

\section{References}

[1] CMS collaboration, Search for lepton-flavour-violating decays of the Higgs boson, Phys. Lett. B 749 (2015) 337 [arXiv: 1502.07400] [INSPIRE].

[2] ATLAS collaboration, Search for lepton-flavour-violating $H \rightarrow \mu \tau$ decays of the Higgs boson with the ATLAS detector, JHEP 11 (2015) 211 [arXiv:1508.03372] [INSPIRE].

[3] G. Blankenburg, J. Ellis and G. Isidori, Flavour-changing decays of a $125 \mathrm{GeV}$ Higgs-like particle, Phys. Lett. B $\mathbf{7 1 2}$ (2012) 386 [arXiv:1202.5704] [INSPIRE].

[4] R. Harnik, J. Kopp and J. Zupan, Flavor violating Higgs decays, JHEP 03 (2013) 026 [arXiv:1209.1397] [INSPIRE].

[5] A. Dery, A. Efrati, Y. Hochberg and Y. Nir, What if $B R(h \rightarrow \mu \mu) / B R(h \rightarrow \tau \tau)$ does not equal $m_{\mu}^{2} / m_{\tau}^{2}$ ?, JHEP 05 (2013) 039 [arXiv: 1302.3229] [INSPIRE].

[6] A. Dery, A. Efrati, Y. Nir, Y. Soreq and V. Susič, Model building for flavor changing Higgs couplings, Phys. Rev. D 90 (2014) 115022 [arXiv:1408.1371] [INSPIRE].

[7] A. Arhrib, Y. Cheng and O.C.W. Kong, Higgs to $\mu+\tau$ decay in supersymmetry without R-parity, Europhys. Lett. 101 (2013) 31003 [arXiv:1208.4669] [INSPIRE].

[8] M. Arroyo, J.L. Diaz-Cruz, E. Diaz and J.A. Orduz-Ducuara, Flavor violating Higgs signals in the texturized two-Higgs doublet model (2HDM-Tx), arXiv:1306.2343 [INSPIRE].

[9] A. Celis, V. Cirigliano and E. Passemar, Lepton flavor violation in the Higgs sector and the role of hadronic $\tau$-lepton decays, Phys. Rev. D 89 (2014) 013008 [arXiv:1309.3564] [INSPIRE].

[10] A. Falkowski, D.M. Straub and A. Vicente, Vector-like leptons: Higgs decays and collider phenomenology, JHEP 05 (2014) 092 [arXiv: 1312.5329] [INSPIRE].

[11] M.D. Campos, A.E.C. Hernández, H. Päs and E. Schumacher, Higgs $\rightarrow \mu \tau$ as an indication for $S_{4}$ flavor symmetry, Phys. Rev. D 91 (2015) 116011 [arXiv:1408.1652] [InSPIRE].

[12] D. Aristizabal Sierra and A. Vicente, Explaining the CMS Higgs flavor violating decay excess, Phys. Rev. D 90 (2014) 115004 [arXiv:1409.7690] [INSPIRE].

[13] J. Heeck, M. Holthausen, W. Rodejohann and Y. Shimizu, Higgs $\rightarrow \mu \tau$ in Abelian and non-Abelian flavor symmetry models, Nucl. Phys. B 896 (2015) 281 [arXiv:1412.3671] [INSPIRE].

[14] A. Crivellin, G. D'Ambrosio and J. Heeck, Explaining $h \rightarrow \mu^{ \pm} \tau^{\mp}, B \rightarrow K^{*} \mu^{+} \mu^{-}$and $B \rightarrow K \mu^{+} \mu^{-} / B \rightarrow K e^{+} e^{-}$in a two-Higgs-doublet model with gauged $L_{\mu}-L_{\tau}$, Phys. Rev. Lett. 114 (2015) 151801 [arXiv: 1501.00993] [INSPIRE].

[15] I. Doršner, S. Fajfer, A. Greljo, J.F. Kamenik, N. Košnik and I. Nišandžic, New physics models facing lepton flavor violating Higgs decays at the percent level, JHEP 06 (2015) 108 [arXiv: 1502.07784] [INSPIRE].

[16] D. Das and A. Kundu, Two hidden scalars around $125 \mathrm{GeV}$ and $h \rightarrow \mu \tau$, Phys. Rev. D 92 (2015) 015009 [arXiv:1504.01125] [INSPIRE]. 
[17] F. Bishara, J. Brod, P. Uttayarat and J. Zupan, Nonstandard Yukawa couplings and Higgs portal dark matter, JHEP 01 (2016) 010 [arXiv:1504.04022] [INSPIRE].

[18] B. Bhattacherjee, S. Chakraborty and S. Mukherjee, $H \rightarrow \tau \mu$ and excess in $t \bar{t} H$ : connecting the dots in the hope for the first glimpse of BSM Higgs signal, arXiv:1505.02688 [INSPIRE].

[19] X.-G. He, J. Tandean and Y.-J. Zheng, Higgs decay $h \rightarrow \mu \tau$ with minimal flavor violation, JHEP 09 (2015) 093 [arXiv: 1507.02673] [INSPIRE].

[20] W. Altmannshofer, S. Gori, A.L. Kagan, L. Silvestrini and J. Zupan, Uncovering mass generation through Higgs flavor violation, Phys. Rev. D 93 (2016) 031301 [arXiv: 1507.07927] [INSPIRE].

[21] K. Cheung, W.-Y. Keung and P.-Y. Tseng, Leptoquark induced rare decay amplitudes $h \rightarrow \tau^{\mp} \mu^{ \pm}$and $\tau \rightarrow \mu \gamma$, Phys. Rev. D 93 (2016) 015010 [arXiv: 1508.01897] [inSPIRE].

[22] E. Arganda, M.J. Herrero, X. Marcano and C. Weiland, Enhancement of the lepton flavor violating Higgs boson decay rates from SUSY loops in the inverse seesaw model, Phys. Rev. D 93 (2016) 055010 [arXiv: 1508.04623] [INSPIRE].

[23] F.J. Botella, G.C. Branco, M. Nebot and M.N. Rebelo, Flavour changing Higgs couplings in a class of two Higgs doublet models, Eur. Phys. J. C 76 (2016) 161 [arXiv:1508.05101] [INSPIRE].

[24] S. Baek and K. Nishiwaki, Leptoquark explanation of $h \rightarrow \mu \tau$ and muon $(g-2)$, Phys. Rev. D 93 (2016) 015002 [arXiv: 1509.07410] [INSPIRE].

[25] A. Brignole and A. Rossi, Lepton flavor violating decays of supersymmetric Higgs bosons, Phys. Lett. B 566 (2003) 217 [hep-ph/0304081] [INSPIRE].

[26] A. Brignole and A. Rossi, Anatomy and phenomenology of $\mu-\tau$ lepton flavor violation in the MSSM, Nucl. Phys. B 701 (2004) 3 [hep-ph/0404211] [INSPIRE].

[27] E. Arganda, A.M. Curiel, M.J. Herrero and D. Temes, Lepton flavor violating Higgs boson decays from massive seesaw neutrinos, Phys. Rev. D 71 (2005) 035011 [hep-ph/0407302] [INSPIRE].

[28] M. Arana-Catania, E. Arganda and M.J. Herrero, Non-decoupling SUSY in LFV Higgs decays: a window to new physics at the LHC, JHEP 09 (2013) 160 [Erratum ibid. 10 (2015) 192] [arXiv: 1304.3371] [INSPIRE].

[29] J. Rosiek, Complete set of Feynman rules for the MSSM: erratum, hep-ph/9511250 [INSPIRE].

[30] M. Papucci, J.T. Ruderman and A. Weiler, Natural SUSY endures, JHEP 09 (2012) 035 [arXiv: 1110.6926] [INSPIRE].

[31] CMS collaboration, Search for a standard model-like Higgs boson in the $\mu^{+} \mu^{-}$and $e^{+} e^{-}$ decay channels at the LHC, Phys. Lett. B 744 (2015) 184 [arXiv:1410.6679] [INSPIRE].

[32] ATLAS collaboration, Search for the standard model Higgs boson decay to $\mu^{+} \mu^{-}$with the ATLAS detector, Phys. Lett. B 738 (2014) 68 [arXiv:1406.7663] [INSPIRE].

[33] R. Rattazzi and U. Sarid, The unified minimal supersymmetric model with large Yukawa couplings, Phys. Rev. D 53 (1996) 1553 [hep-ph/9505428] [INSPIRE].

[34] J. Hisano and S. Sugiyama, Charge-breaking constraints on left-right mixing of stau's, Phys. Lett. B 696 (2011) 92 [Erratum ibid. B 719 (2013) 472] [arXiv: 1011.0260] [INSPIRE]. 
[35] W. Altmannshofer, M. Carena, N.R. Shah and F. Yu, Indirect probes of the MSSM after the Higgs discovery, JHEP 01 (2013) 160 [arXiv:1211.1976] [INSPIRE].

[36] M. Carena, S. Gori, I. Low, N.R. Shah and C.E.M. Wagner, Vacuum stability and Higgs diphoton decays in the MSSM, JHEP 02 (2013) 114 [arXiv:1211.6136] [INSPIRE].

[37] W. Altmannshofer, C. Frugiuele and R. Harnik, Fermion hierarchy from sfermion anarchy, JHEP 12 (2014) 180 [arXiv: 1409.2522] [INSPIRE].

[38] E. Arganda, M.J. Herrero, R. Morales and A. Szynkman, Analysis of the $h, H, A \rightarrow \tau \mu$ decays induced from SUSY loops within the mass insertion approximation, JHEP 03 (2016) 055 [arXiv: 1510.04685] [INSPIRE].

[39] N. Craig, F. D'Eramo, P. Draper, S. Thomas and H. Zhang, The hunt for the rest of the Higgs bosons, JHEP 06 (2015) 137 [arXiv: 1504.04630] [INSPIRE].

[40] Belle collaboration, K. Hayasaka et al., New search for $\tau \rightarrow \mu \gamma$ and $\tau \rightarrow e \gamma$ decays at Belle, Phys. Lett. B 666 (2008) 16 [arXiv:0705.0650] [INSPIRE].

[41] Particle Data Group collaboration, K.A. Olive et al., Review of particle physics, Chin. Phys. C 38 (2014) 090001 [InSPIRE]. 